\title{
BET bromodomain proteins regulate transcriptional reprogramming in genetic dilated cardiomyopathy
}

\author{
Andrew Antolic, ${ }^{1}$ Hiroko Wakimoto, ${ }^{2}$ Zhe Jiao, ${ }^{1}$ Joshua M. Gorham, ${ }^{2}$ Steven R. DePalma, ${ }^{2}$ \\ Madeleine E. Lemieux, ${ }^{3}$ David A. Conner, ${ }^{2}$ Da Young Lee, ${ }^{1}$ Jun $Q \mathbf{Q},{ }^{3}$ Jonathan G. Seidman, ${ }^{2}$ \\ James E. Bradner, ${ }^{4,5}$ Jonathan D. Brown, ${ }^{6}$ Saptarsi M. Haldar, ${ }^{7,89}$ Christine E. Seidman, ${ }^{2,4,10}$ \\ and Michael A. Burke' \\ 'Emory University School of Medicine, Atlanta, Georgia, USA. ${ }^{2}$ Harvard Medical School, Boston, Massachusetts, USA \\ ${ }^{3}$ Bioinfo, Plantagenet, Ontario, Canada. ${ }^{4}$ Dana-Farber Cancer Institute, Boston, Massachusetts, USA. ${ }^{5}$ Brigham and \\ Women's Hospital, Boston, Massachusetts, USA. ${ }^{6}$ Vanderbilt School of Medicine, Nashville, Tennessee, USA. ${ }^{7} \mathrm{Gladstone}$ \\ Institute of Cardiovascular Disease, San Francisco, California, USA. ${ }^{8}$ Department of Medicine, Cardiology Division, UCSF \\ School of Medicine, San Francisco, California, USA. ${ }^{9}$ Amgen Research, South San Francisco, California, USA. ${ }^{10}$ Howard \\ Hughes Medical Institute.
}

The bromodomain and extraterminal (BET) family comprises epigenetic reader proteins that are important regulators of inflammatory and hypertrophic gene expression in the heart. We previously identified the activation of proinflammatory gene networks as a key early driver of dilated cardiomyopathy (DCM) in transgenic mice expressing a mutant form of phospholamban $\left(P L N^{R 9 C}\right.$ ) - a genetic cause of DCM in humans. We hypothesized that BETs coactivate this inflammatory process, representing a critical node in the progression of DCM. To test this hypothesis, we treated PLN ${ }^{R 9 C}$ or age-matched WT mice longitudinally with the small molecule BET bromodomain inhibitor JQ1 or vehicle. BET inhibition abrogated adverse cardiac remodeling, reduced cardiac fibrosis, and prolonged survival in $\mathrm{PLN}^{\mathrm{RgC}}$ mice by inhibiting expression of proinflammatory gene networks at all stages of disease. Specifically, JQ1 had profound effects on proinflammatory gene network expression in cardiac fibroblasts, while having little effect on gene expression in cardiomyocytes. Cardiac fibroblast proliferation was also substantially reduced by JQ1. Mechanistically, we demonstrated that BRD4 serves as a direct and essential regulator of NF- $\kappa B-m e d i a t e d$ proinflammatory gene expression in cardiac fibroblasts. Suppressing proinflammatory gene expression via BET bromodomain inhibition could be a novel therapeutic strategy for chronic DCM in humans.

Conflict of interest: JCS and CES are founders and own shares in MyoKardia Inc., a startup company that is developing therapeutics that target the sarcomere. SMH is an executive, officer, and shareholder of Amgen; and a founder of Tenaya Therapeutics with ownership stake. JEB is president of the Novartis Institutes for Biomedical Research and has ownership interest (including stocks, patents, etc.) in Novartis.

Copyright: (c) 2020, American Society for Clinical Investigation.

Submitted: April 3, 2020

Accepted: June 17, 2020

Published: August 6, 2020.

Reference information: JCI Insight. 2020;5(15):e138687.

https://doi.org/10.1172/jci.

insight.138687.

\section{Introduction}

Heart failure (HF) is a common and morbid disease. Existing pharmacotherapies have improved clinical outcomes for HF patients, but residual mortality remains exceedingly high, with an estimated 5-year survival of approximately 50\% (1). HF often results from dilated cardiomyopathy (DCM), a heterogeneous disease with a strong genetic basis (2). Characteristic phenotypic features of DCM include ventricular wall thinning, cardiac chamber dilatation, eccentric myocyte hypertrophy, diffuse interstitial fibrosis, and reduced systolic function. Concomitant with this negative cardiac remodeling is the activation of a suite of cardiac signaling cascades that result in pathologic gene expression. These dynamic changes in gene expression are coordinately controlled in temporal fashion at different stages of disease (3).

To study the regulation of pathologic gene expression in DCM, we used a transgenic mouse model expressing a missense mutation (p.Arg9Cys) in phospholamban $\left(\mathrm{PLN}^{\mathrm{R} 9 \mathrm{C}}\right)$, a known genetic cause of human DCM. In the heart, phospholamban controls calcium cycling via regulation of the cardiac sarcoplasmic/ endoplasmic reticulum calcium adenosine triphosphate (SERCA2a) pump (4). Abnormal cardiomyocyte calcium homeostasis is a common and proximal mediator of stress-induced cardiac remodeling $(5,6)$. PLN ${ }^{\mathrm{R} 9 \mathrm{C}}$ mice display altered calcium handling before onset of phenotypic disease (preDCM) and ultimately develop progressive DCM with fulminant heart failure culminating in premature death (4). This inexorable process 
mimics the disease course of individuals carrying this mutation $(4,7,8)$ and recapitulates the natural history of DCM. Thus, $\mathrm{PLN}^{\mathrm{R} 9 \mathrm{C}}$ is a robust model by which to study the molecular changes that underlie DCM.

We previously demonstrated temporal changes in gene expression in $\mathrm{PLN}^{\mathrm{R} 9 \mathrm{C}}$ hearts (3). These findings were similar to those seen in human induced pluripotent stem cells engineered to carry the PLN ${ }^{\mathrm{R} 9 \mathrm{C}}$ mutation (9), and highlight the coordinated integration of multiple signaling networks into a concentrated and temporal stress response program. Pathologic gene networks activated in $\mathrm{HF}$ are also associated with dynamic remodeling of chromatin $(10,11)$, including changes in lysine acetylation of histone amino-terminal tails. These acetylated lysine residues are recognized by epigenetic reader proteins that contain acetyl-lysine recognition domains (bromodomains), which in turn regulate gene transcription. The bromodomain and extraterminal (BET) family (including BRD2, BRD3, and BRD4, which are expressed in the heart) bind to acetylated chromatin via their dual $\mathrm{N}$-terminal bromodomains. Upon binding to acetyl-lysine residues, BRD4 coactivates gene transcription by assembling protein complexes that promote pause release of RNA polymerase $(12,13)$. BETs have been identified as critical coactivators of pathologic gene expression in rodent models of pressure overload, agonist-induced and ischemia-mediated heart failure, and BET inhibition exerts therapeutic effects via preferential suppression of inflammatory and profibrotic transcriptional programs (13-15).

The PLN ${ }^{\mathrm{R} 9 \mathrm{C}}$ mouse model features robust activation of a broad array of inflammatory gene networks very early in the disease course, before the onset of left ventricle (LV) dilation or systolic dysfunction (3). However, it remains unknown whether activation of this transcriptional program plays a causal role in DCM pathogenesis. Interestingly, the genes and networks upregulated in PLN ${ }^{\mathrm{R} 9 \mathrm{C}}$ mice were remarkably similar to those suppressed by JQ1 treatment in other mouse cardiomyopathy models. Therefore, we tested the hypothesis that inhibition of BETs with the small molecule JQ1 could directly suppress pathologic inflammatory gene expression and thereby exert salutary effects in this model of chronic and insidiously progressive genetic DCM. Herein, we demonstrate the effectiveness of JQ1 in suppressing inflammatory gene network activation, thus establishing a causal relationship between early pathologic inflammatory gene expression and DCM progression. We identify cardiac fibroblasts as a key cell type driving this process, and define a critical mechanistic link between BRD4 and NF- $\mathrm{KB}$. These data demonstrate that BETs serve as essential nodal activators of cardiac fibroblasts in DCM.

\section{Results}

Genes downregulated by BET inhibition are highly enriched in $P L N^{R 9 C}$ hearts. $\mathrm{PLN}^{\mathrm{R} 9 \mathrm{C}}$ mice display robust inflammatory and profibrotic gene network activation before there is any phenotypic evidence of DCM (preDCM), but it is not clear whether this plays a causal role in DCM progression (3). BET inhibition with JQ1 suppresses this inflammatory program in other models of cardiomyopathy, including thoracic aortic constriction (TAC), agonist-induced and myocardial infarction (MI) (13-15). Using gene set enrichment analysis (GSEA), we identified a significant positive enrichment for genes induced in PLN ${ }^{\mathrm{R} C}$ hearts when compared with a manually curated set of known BET-regulated genes identified in hearts from both TAC and MI models (Figure 1) $(3,15)$. Gene ontology (GO) analysis has previously revealed that inflammatory pathways are robustly suppressed by JQ1 in TAC and MI (15); these same pathways were also highly enriched in PLN ${ }^{\mathrm{R} 9 \mathrm{C}}$ hearts (Table 1) (3). Finally, we noted that BET expression was robust in $\mathrm{PLN}^{\mathrm{R} 9 \mathrm{C}}$ hearts (Supplemental Figure 1; supplemental material available online with this article; https://doi.org/10.1172/jci.insight.138687DS1). These data provided a strong rationale for us to test whether BET inhibition could suppress these pathologic inflammatory gene expression programs in $\mathrm{PLN}^{\mathrm{R} 9 \mathrm{C}}$ mice and thereby halt initiation or progression of DCM.

BET inhibition blunts negative cardiac remodeling and improves survival in PLN ${ }^{R 9 C}$. We injected PLN ${ }^{\mathrm{R} 9 \mathrm{C}}$ or age-matched WT mice with the BET bromodomain inhibitor JQ1 at $50 \mathrm{mg} / \mathrm{kg}$ or vehicle (Figure $2 \mathrm{~A}$ ). We first assessed the effects of BET inhibition on cardiac structure and function. Treatment of WT mice with JQ1 had no significant effect on cardiac structure or function (Figure 2, B-F). PLN ${ }^{\mathrm{R} 9 \mathrm{C}}$ vehicletreated mice demonstrated progressive negative cardiac remodeling, as evidenced by reduced fractional shortening (FS), increased LV end systolic diameter (LVESD), increased LV end diastolic diameter (LVEDD), and decreased LV wall thickness (LVWT; $P<0.001$ for all) compared with WT vehicle-treated mice (Figure 2, C-F). By contrast, JQ1-treated PLN ${ }^{\mathrm{R} 9 \mathrm{C}}$ mice had better LV systolic function (smaller LVESD and higher FS; $P<0.001$ ) and less LV remodeling (smaller LVEDD and greater LVWT; $P<0.001)$ than $\mathrm{PLN}^{\mathrm{R} 9 \mathrm{C}}$ vehicle-treated mice. Compared with $\mathrm{WT}$ vehicle-treated mice, $\mathrm{PLN}{ }^{\mathrm{R} 9 \mathrm{C}} \mathrm{JQ} 1$ treated mice still showed lower FS $(P<0.001)$ but did not differ significantly from WT mice in LVESD $(P=0.04), \operatorname{LVEDD}(P=0.51)$, or LVWT $(P=0.03)$ at 20 weeks of age. Similarly, heart weight to body 
A

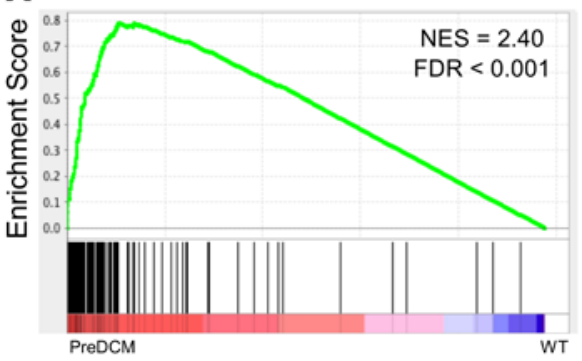

B

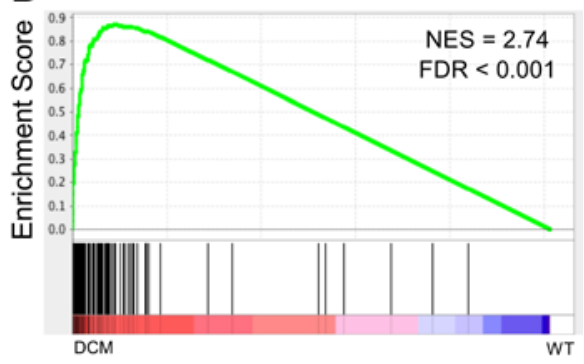

Figure 1. Genes suppressed by JQ1 are highly enriched in PLN ${ }^{\mathrm{RgC}}$ hearts. GSEA for preDCM (A) and DCM (B) PLN ${ }^{\mathrm{RgC}}$ mice against genes that were upregulated in both TAC and MI models and were downregulated by JQ1 treatment in both (15). NES, normalized enrichment score.

Table 1. Enriched JQ1-responsive Gene Ontology terms in PLN ${ }^{\mathrm{RgC}}$ DCM hearts

\begin{tabular}{lcc}
\hline GO term & $\begin{array}{c}\text { Reduced by JQ1 in TAC and MI } \\
\text { (extracted from ref. 15), FDR }\end{array}$ & $\begin{array}{c}\text { Increased in PLN } \\
\text { from DCM (extracted }\end{array}$ \\
$\begin{array}{l}\text { CO:0006955, immune response } \\
\text { G0:0006952, defense response }\end{array}$ & $<1 \times 10^{-11}$ & $1 \times 10^{-15}$ \\
$\begin{array}{l}\text { CO:0045087, innate immune } \\
\text { response }\end{array}$ & $<1 \times 10^{-5}$ & $2 \times 10^{-17}$ \\
$\begin{array}{l}\text { G0:0007155, cell adhesion } \\
\text { G0:0034097, response to cytokine }\end{array}$ & $<1 \times 10^{-4}$ & $2 \times 10^{-4}$ \\
CO, Gene Ontology. & $<1 \times 10^{-3}$ & $1 \times 10^{-27}$ \\
\hline
\end{tabular}

weight ratio was significantly higher in PLN ${ }^{\mathrm{R} 9 \mathrm{C}}$ vehicle-treated mice compared with $\mathrm{WT}(P<0.001)$, and returned to normal levels with JQ1 (Supplemental Figure 2).

$\operatorname{PLN}^{\mathrm{R} 9 C}$ mice also develop substantial fibrosis with progression to DCM $(3,4)$. To assess whether JQ1 could attenuate this response, we stained hearts from 20-week-old mice with Masson's trichrome, and quantified LV fibrosis (Figure 2, G-I). WT mice did not develop fibrosis ( $0.3 \%$ of LV area). PLN ${ }^{\mathrm{R} 9 \mathrm{C}}$ vehicle-treated mice displayed severe fibrosis ( $18.4 \% \pm 0.7 \% ; P<0.001$ vs. WT vehicle-treated). JQ1 significantly reduced the amount of $\mathrm{LV}$ fibrosis, by a factor of 3.2 -fold $\left(5.8 \% \pm 0.4 \%, P<0.001\right.$ vs. PLN $^{\mathrm{R} 9 \mathrm{C}}$ vehicle-treated).

A separate cohort of mice $(n=16)$ was followed for survival (Figure 3). Mice were treated with JQ1 or vehicle beginning at 8 weeks of age and monitored for mortality (as defined by our IACUC protocol; see Methods). JQ1 treatment increased lifespan by 2.5 weeks $(10.1 \%)$ in $\operatorname{PLN}^{\mathrm{R} 9 \mathrm{C}}$ mice $(P<0.001)$. The average survival of PLN ${ }^{\mathrm{R} 9 \mathrm{C}}$ vehicle-treated mice was the same as that of a contemporary cohort of untreated PLN ${ }^{\mathrm{R} 9 \mathrm{C}}$ mice (24.9 \pm 1.8 vs. $23.1 \pm 4.3$ weeks of age; log-rank, $P=0.37$ ). These data demonstrate that JQ1 substantially delays disease progression and conveys a survival advantage in a model of chronic, severe, and progressive DCM.

BETs control a pathologic gene expression program. Next, we assessed the primary gene regulatory effects of BET inhibition at a very early stage of disease. We performed RNA-Seq on whole LV tissue from PLN ${ }^{\mathrm{R} 9}$ mice at the preDCM stage and age-matched WT controls treated with JQ1 or vehicle (Figure 4A and Supplemental Table 1). Principal component analysis (PCA) demonstrated a pattern of gene expression in PLN${ }^{\mathrm{R} 9 \mathrm{C}}$ vehicle-treated preDCM mice that was clearly distinct from those of the other groups, whereas JQ1 treatment normalized gene expression in PLN ${ }^{\mathrm{R} 9 \mathrm{C}}$ mice (Supplemental Figure 3). In WT mice, BET inhibition resulted in differential expression of 692 genes (244 upregulated, 448 downregulated). However, no specific pathways were significantly enriched after correction for multiple hypothesis testing (Supplemental Table 2).

PreDCM PLN ${ }^{\mathrm{R} 9 \mathrm{C}}$ vehicle-treated hearts were heavily enriched for proinflammatory genes and pathways (Supplemental Tables 1 and 2). Nearly all differentially expressed genes in PLN ${ }^{R 9 C}$ vehicle-treated hearts were upregulated (Figure 4B). Stress response genes (the natriuretic peptides, fetal myosin heavy chain, growth differentiation factor 15 ) were markedly increased in preDCM PLN ${ }^{\mathrm{R} 9 \mathrm{C}}$ vehicletreated hearts, as were genes involved in TGF- $\beta$ signaling (Tgfb2, Tgfb3, Tgfbr2, Ctgff) and the innate immune response (Tlr2, Tlr4; Supplemental Figure 4A). The pattern of differentially expressed genes in PLN ${ }^{R 9 C}$ 
A Studie
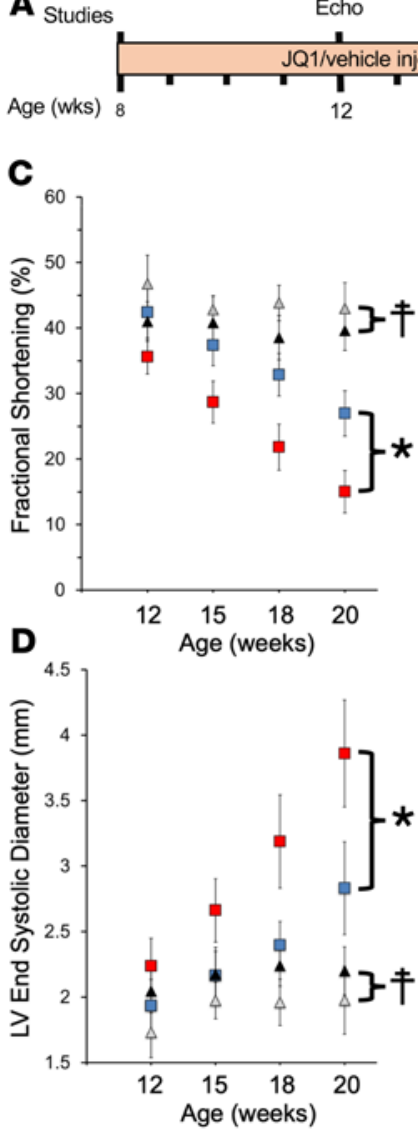

$\square$ PLN JQ1

$\triangle$ WT JQ1

$\square$ PLN vehicle

$\Delta$ WT vehicle
Echo Echo Echo/harvest
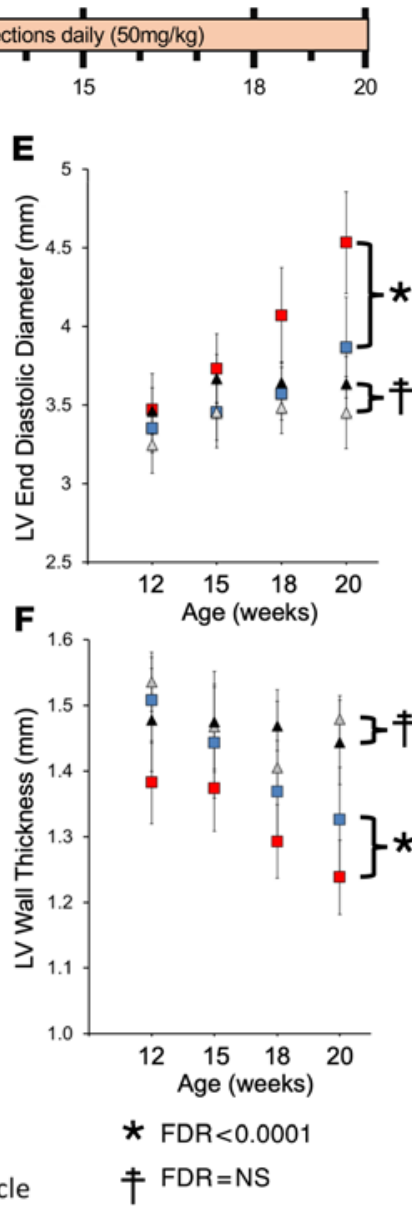

B
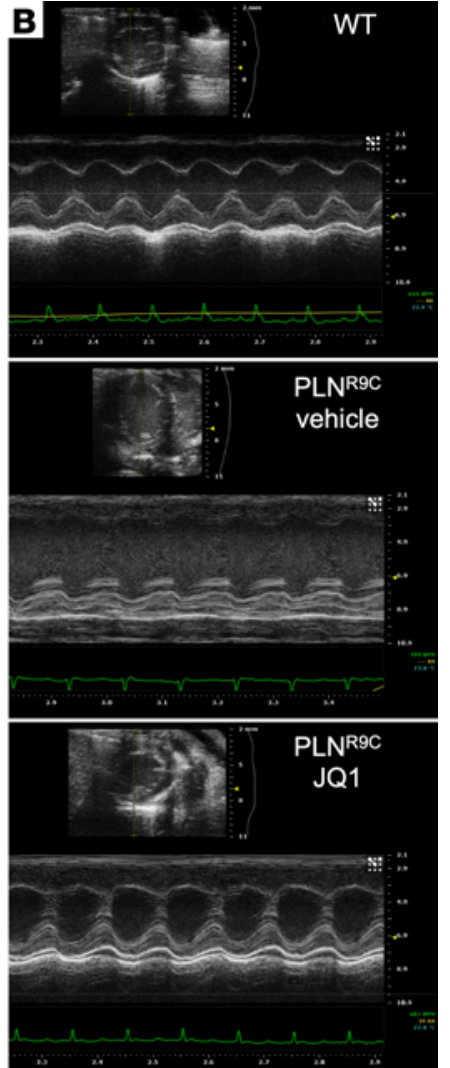

G

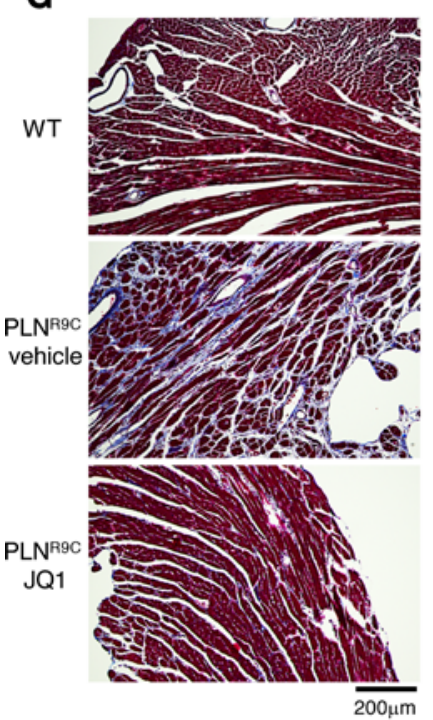

H
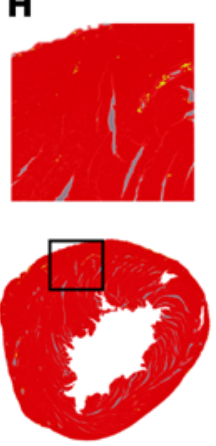

WT
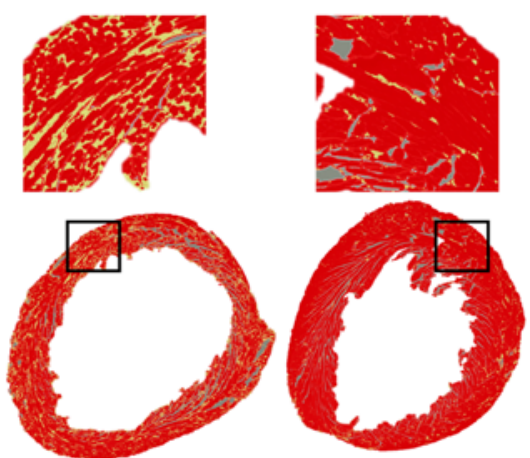

PLNR9C JQ1

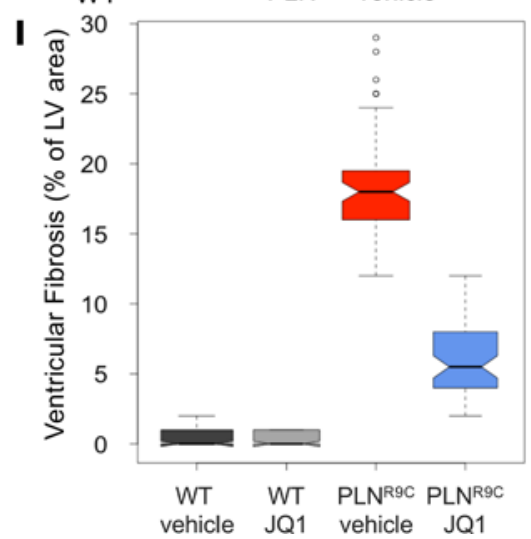

$\triangle W T$ JQ1

$\Delta$ WT vehicle

$\square$ PLN JQ1

$\square$ PLN vehicle
Figure 2. BET inhibition delays DCM in PLN ${ }^{\mathrm{RgC}}$ mice.

(A) Experimental protocol. (B) Representative M-mode images of WT and PLN ${ }^{R 9 C}$ vehicle- or JQ1-treated mice at 18 weeks of age. Echo, echocardiography. (C-F) Echocardiographic assessment of mice treated with JQ1 or vehicle demonstrates progressive systolic dysfunction and negative LV remodeling in $P L N^{R 9 C}$ mice that was significantly blunted by JQ1 ( $n=14$ PLN $^{\text {R9C }}, n$ = $7 \mathrm{WT}$ mice per group; ANOVA corrected for multiple hypothesis testing). Representative images demonstrating cardiac fibrosis: (C) Masson's trichrome-stained LV sections from WT and PLN ${ }^{R 9 C}$ hearts and $(\mathbf{H})$ false-color images of fibrosis identified by Keyence microscope software (yellow, fibrosis; box, enlarged region). As JQ1 had no effect on fibrosis in WT, a single representative WT image is shown. Scale bar in $\mathbf{G}$ : $200 \mu \mathrm{m}$. Original magnification in $\mathbf{H}, \times 10$. (I) Quantification of scar area demonstrated severe fibrosis in PLN ${ }^{R 9 C}$ vehicle-treated hearts at 20-weeks of age that was markedly blunted by JQ1 ( $n=3$ mice, 36 images from $n=4$ levels from apex to base for each mouse). Boxes, IQR; whiskers, 1.5× IQR black line, median; notches, SD, circles, extreme outlier values). 


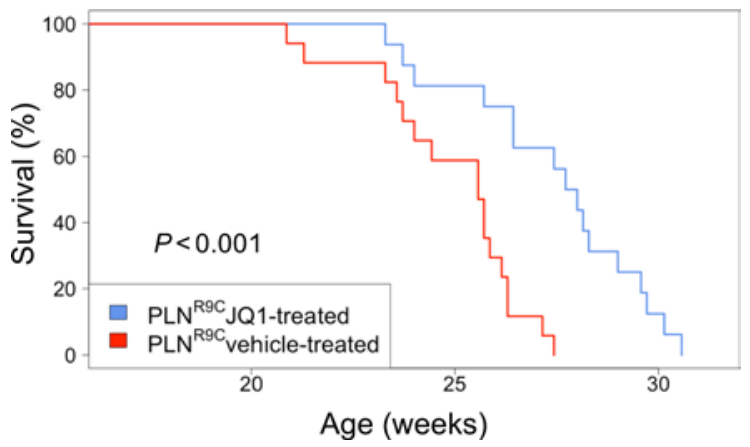

Figure 3. BET inhibition prolongs survival. Kaplan-Meier plot of survival in PLN ${ }^{R 9 C}$ mice treated with vehicle or JQ1 ( $n=16$ mice/treatment group, log-rank test).

vehicle-treated mice predicted activation of key inflammatory pathways and proinflammatory transcriptional regulators, implicating TGF- $\beta, \mathrm{NF}-\mathrm{\kappa B}, \mathrm{AKT}$, and both the innate and adaptive immune response systems as major early effectors of DCM (Figure 4C, Supplemental Figure 4, A and B, and Supplemental Table 3). GSEA revealed that genes downregulated by JQ1 in TAC and MI models matched those enriched in $\mathrm{PLN}^{\mathrm{R} 9 \mathrm{C}}$ vehicle-treated hearts (Supplemental Figure 4C).

Treatment with JQ1 strongly attenuated these gene expression changes (Figure 4B). Relative to vehicle-treated PLN ${ }^{\mathrm{R} 9 \mathrm{C}}$ mice, JQ1 reduced the expression of 663 genes $(80.1 \%$ of differentially expressed genes). Pathway analysis and GSEA revealed near-uniform suppression of these inflammatory gene networks and proinflammatory upstream regulators (i.e., TGF- $\beta$, TLRs, cytokines) by JQ1 in preDCM PLN ${ }^{\mathrm{R} 9 \mathrm{C}}$ hearts, matching the effects of JQ1 in TAC and MI models (Figure 4D and Supplemental Figure 4, B, D, and E). These data demonstrate that BETs strongly and specifically regulate inflammatory gene network activation in preDCM LV tissue, a stage at which changes in LV geometry, systolic function, or fibrosis have yet to manifest.

ChronicBETinhibition dampenscardiacinflammatorysignaling. To study the effects of long-term BET inhibition, we treated mice from 8 to 20 weeks of age with JQ1 or vehicle and performed RNA-Seq on whole LV tissue(Figure 5A).PCA again demonstrated cleargrouping of mice, with a shift toward WT gene expression in $P L N^{R 9 C}$ JQ1-treated hearts (Supplemental Figure 5). LN $^{\mathrm{R} 9 \mathrm{C}}$ vehicle-treated mice with overt DCM demonstrated progressive inflammatory gene network activation (Supplemental Tables 4-6). At this later stage of disease, we also identified a concomitant shift in metabolic gene expression (Figure 5, B and $\mathrm{C}$ ), consistent with our prior studies (3). There was near uniform reduction in the expression of aerobic metabolic genes, including those involved in mitochondrial oxidative phosphorylation, fatty acid oxidation, and the tricarboxylic acid cycle, with concurrent increases in expression of most genes controlling cellular glucose utilization.

Longitudinal treatment with JQ1 durably suppressed pathologic inflammatory gene expression, though the effect on metabolic gene expression was only partial at this late stage of disease. Pathways suppressed included fibrosis and Wnt signaling networks (Table 2). While many genes and pathways were still dysregulated in PLN ${ }^{\mathrm{R} 9 \mathrm{C}} \mathrm{JQ} 1$-treated mice compared with WT, the magnitude of effect remained substantially lower than that observed in $\mathrm{PLN}^{\mathrm{R} 9 \mathrm{C}}$ vehicle-treated mice, demonstrating the continued effect of JQ1 in late-stage DCM (Figure 5, D and E). Collectively, these data show that BET inhibition beginning in preDCM mice potently and specifically suppressed pathologic inflammatory and profibrotic gene networks longitudinally, leading to substantially reduced myocardial fibrosis and blunted negative cardiac remodeling, thus establishing a causal role for these inflammatory gene networks in DCM progression.

BET inhibition suppresses fibroblast activation. Given the salutary effects on inflammatory gene network expression and fibrosis, we tested whether BET inhibition had differential effects in the different cardiac cell compartments. We previously identified excess non-myocyte proliferation in PLN ${ }^{\mathrm{R} 9 \mathrm{C}}$ mice (3). BETs have been shown to regulate cell proliferation in multiple other contexts (16-19). To test whether BETs play a role in the proliferation of non-myocytes in this model, PLNR9C and WT mice treated with JQ1 or vehicle were injected with 5-ethynyl-2'-deoxyuracil (EdU) before sacrifice (Figure 6A). Proliferating non-myocytes were 3.7-fold more prevalent $(P<0.001)$ in $\mathrm{PLN}^{\mathrm{R} 9 \mathrm{C}}$ vehicle-treated mice compared with WT (Figure 6, B and C). This was reduced 1.9-fold by JQ1 $(P<0.001)$. Additionally, our RNA-Seq data showed a strong increase in genes that are characteristic of myofibroblasts 
A

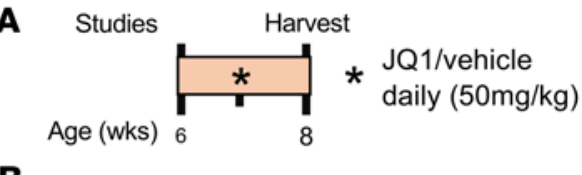

B

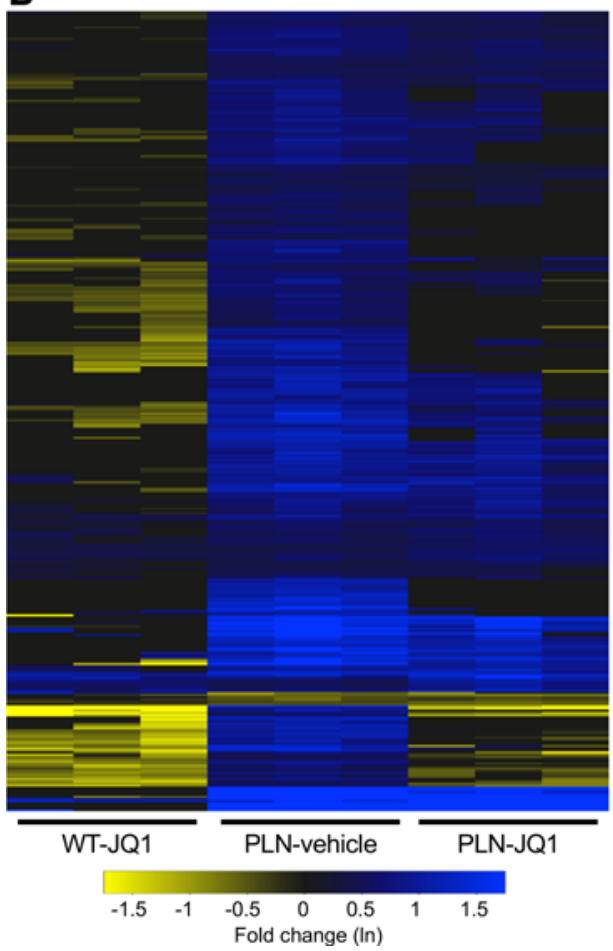

C PLNR9C vehicle vs. WT vehicle

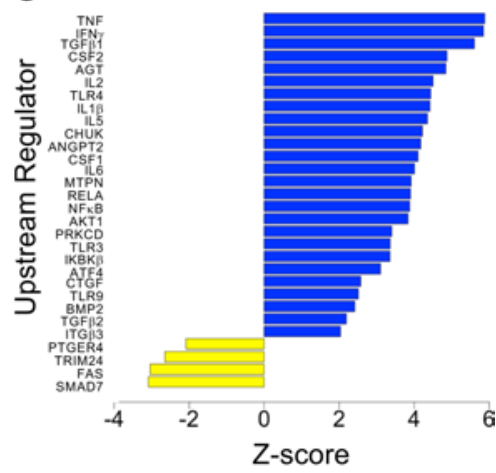

D PLNR9 JQ1 vs. PLNR9C vehicle

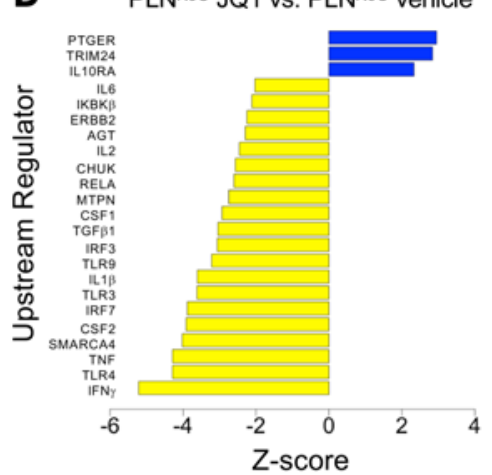

Figure 4. BETs activate inflammatory gene expression in preDCM hearts. (A) Experimental protocol ( $n=3$ mice) treatment group). (B) Heat map of genes significantly differentially expressed in preDCM PLN ${ }^{R 9 C}$ vehicle-treated hearts, demonstrating global reduction in gene activation in PLN ${ }^{R 9 C}$ JQ1-treated mice. Data plotted are natural log (In) of fold change values versus those in WT vehicle-treated mice. (C) IPA upstream regulator analysis in preDCM PLN ${ }^{\mathrm{RgC}}$ vehicle-treated compared with WT mice predicted the activation of many inflammatory mediators. (D) IPA upstream regulator analysis demonstrating reduced activity of the majority of proinflammatory mediators in preDCM PLN ${ }^{R 9 C}$ JQ1-treated versus PLN ${ }^{\mathrm{RSC}}$ vehicle-treated mice.

in DCM hearts; these were reduced by JQ1 (Supplemental Figure 6). Therefore, we hypothesized that these proliferating cells were cardiac fibroblasts.

As there is no single consensus fibroblast marker, we undertook a multimarker histochemical approach to identify the nature of the EdU-positive cells in PLN ${ }^{R 9 C}$ hearts. Vimentin staining in the absence of costaining for CD31 (endothelial cells) and CD45 (myeloid cells) is an accepted means of identifying fibroblasts $(20,21)$. We found that the vast majority $(94.6 \% \pm 1.0 \%)$ of EdU-positive non-myocytes costained with vimentin, with only a small percentage staining with CD31 $(3.7 \% \pm 1.2 \%)$ or CD45 $(1.8 \% \pm 0.4 \%)$, thus confirming that the proliferating cells were predominantly cardiac fibroblasts (Figure 6, D and E). There was no significant difference in the percentage of EdU-positive cells costained with vimentin, CD31, or CD45 in WT or PLN ${ }^{\mathrm{R} 9}$ mice treated with either vehicle or JQ1 (data not shown). We also observed significant decreases in genes associated with myofibroblast activation in DCM mice treated with JQ1 by RNA-Seq, including Postn (-1.9-fold, $P<0.001)$, Eln (-3.0-fold, $P<0.001)$, Lox (-1.7-fold, $P<0.001$ ), Itga 1 $(-1.5$-fold, $P<0.001)$ and $P d g$ fra $(-1.6$-fold, $P<0.001)$. These data affirm the role of BETs in cardiac fibroblast activation in DCM (Supplemental Figure 6).

Next, to test the cell compartment-specific effects of JQ1 on gene expression, we performed RNASeq on isolated pools of cardiac non-myocytes and cardiomyocytes (Figure 7A). JQ1 had a substantial effect on the pattern of gene expression in non-myocytes (Figure 7B), but not in cardiomyocytes (Figure $7 \mathrm{E})$. Similarly, JQ1 drove a significant change in gene expression in PLN ${ }^{\mathrm{R} C \mathrm{C}}$ non-myocytes (Figure 7D) but not cardiomyocytes (Figure 7F). Enriched pathways in PLN ${ }^{R 9 C}$ non-myocytes predominantly consisted of inflammatory gene networks that were activated with progression to DCM, an effect completely 


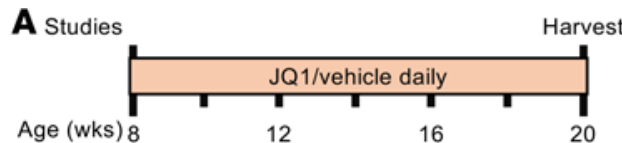

B

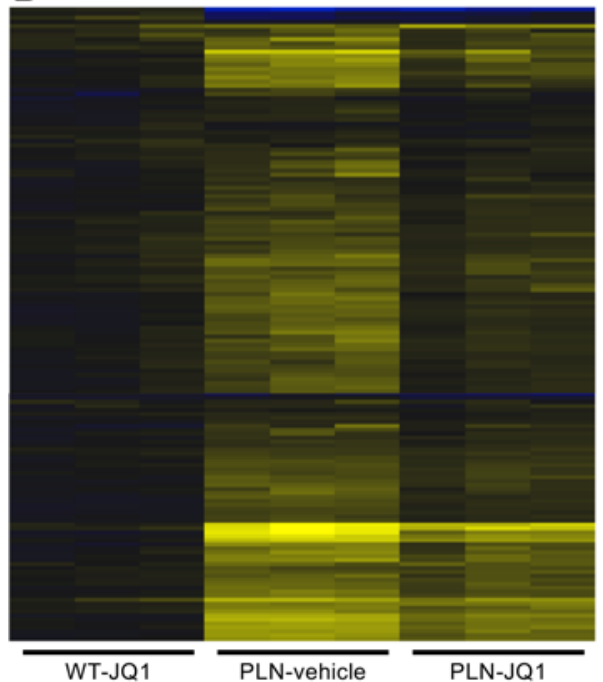

C

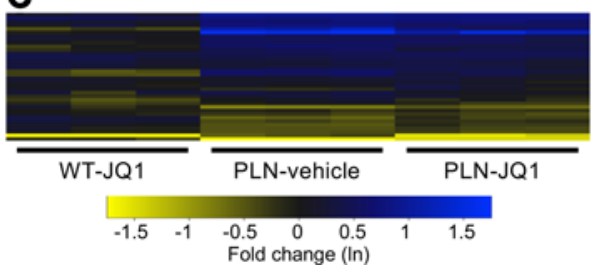

D

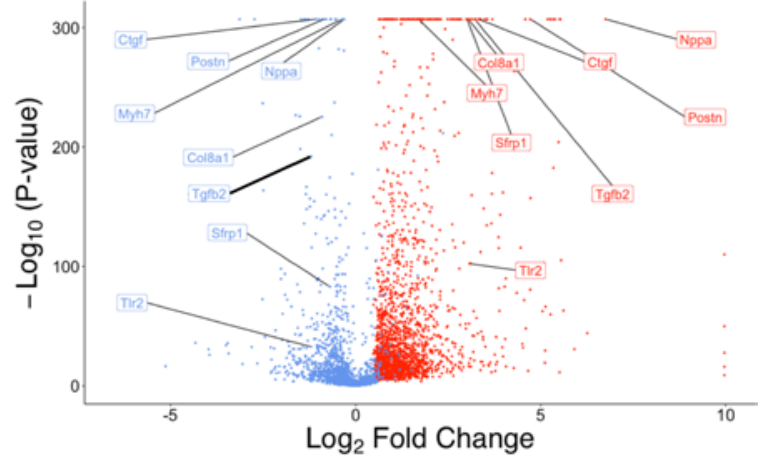

E

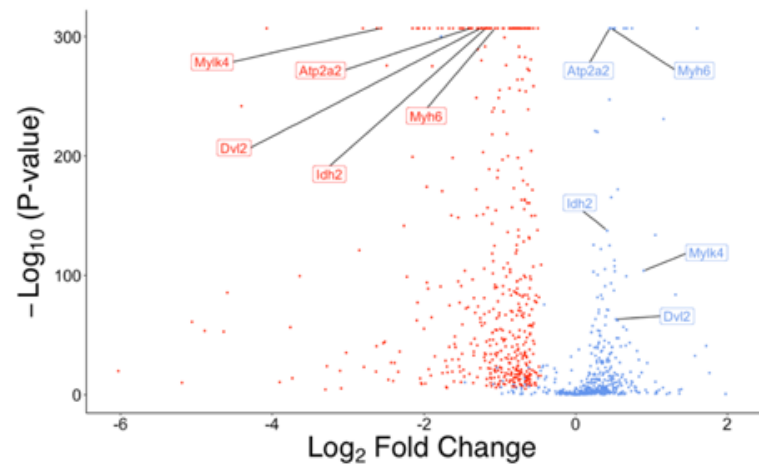

$\square$ PLN JQ1 $\square$ PLN vehicle

Figure 5. Chronic BET inhibition alters inflammatory gene expression in DCM. (A) Experimental protocol ( $n=3$ mice/treatment group). Heat maps demonstrating that (B) genes controlling aerobic respiration (tricarboxylic acid cycle and mitochondrial oxidative phosphorylation) were almost uniformly downregulated, while (C) genes controlling cellular glucose utilization were generally upregulated in PLN ${ }^{R 9 C}$ hearts. JQ1 had little effect on metabolic gene expression. Data plotted are natural log of fold-change values versus WT vehicle-treated mice. Volcano plots demonstrating the magnitude and significance of JQ1's alteration of the expression of genes that were (D) upregulated or (E) downregulated in PLN ${ }^{\mathrm{R} 9 \mathrm{C}}$ vehicle-treated hearts. In each plot, blue represents the effect of JQ1 on gene expression for all genes in red. Representative genes (labeled) reveal key processes affected by JQ1, including cardiac stress response signaling (Nppa, Myh6/Myh7), fibrosis and extracellular matrix remodeling (Postn, Col8a1), TGF- $\beta$ signaling (Tgfb2, Ctgf), cytoskeletal signaling (Mylk4), Wnt signaling (Sfrp1, Dv/2), innate immune activation (T/r2, T/r4), and metabolism (Idh2, Atp2a2).

\section{Table 2. Pathways enriched in PLN ${ }^{R 9 C}$ JQ1- versus vehicle-treated mice}

\begin{tabular}{llc}
\hline Ingenuity canonical pathway & FDR & Differentially expressed genes \\
Hepatic fibrosis/hepatic stellate cell activation & $2 \times 10^{-4}$ & $13 \%$ \\
Colorectal cancer metastasis signaling & $2 \times 10^{-3}$ & $11 \%$ \\
$\begin{array}{l}\text { Role of macrophages, fibroblasts and } \\
\text { endothelial cells in rheumatoid arthritis }\end{array}$ & $2 \times 10^{-3}$ & $10 \%$ \\
Wnt/ $\beta$-catenin signaling & $2 \times 10^{-3}$ & $12 \%$
\end{tabular}

eliminated by JQ1 (Figure 7C and Supplemental Table 7). By contrast, cellular metabolic pathways (the predominant program altered in DCM cardiomyocytes) all remained enriched in PLN ${ }^{\mathrm{R} 9 \mathrm{C}}$ cardiomyocytes after JQ1 treatment (Table 3). Collectively, these data demonstrate that BETs play a critical role in the expression of dynamically regulated inflammatory gene networks in cardiac non-myocytes, and suggest that BET inhibition does not exert its salutary effects on heart function in this model via modulation of adult cardiomyocyte gene expression programs. 
A

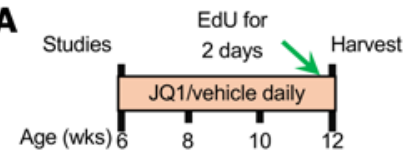

B

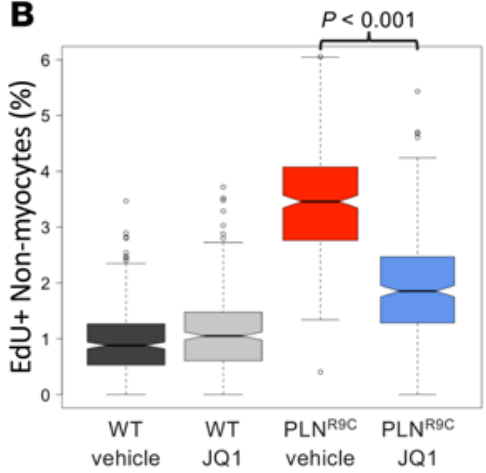

C

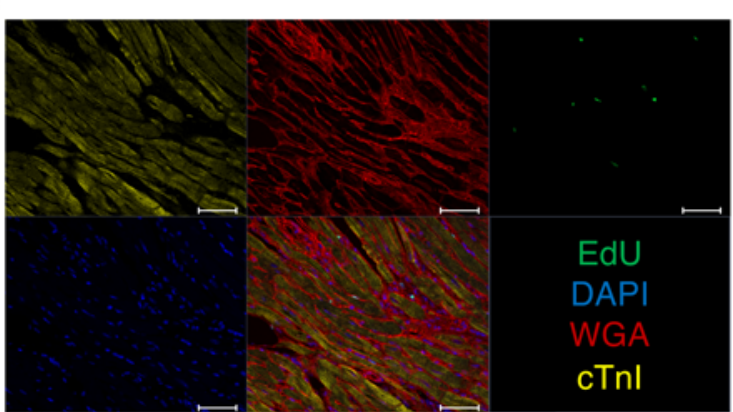

D
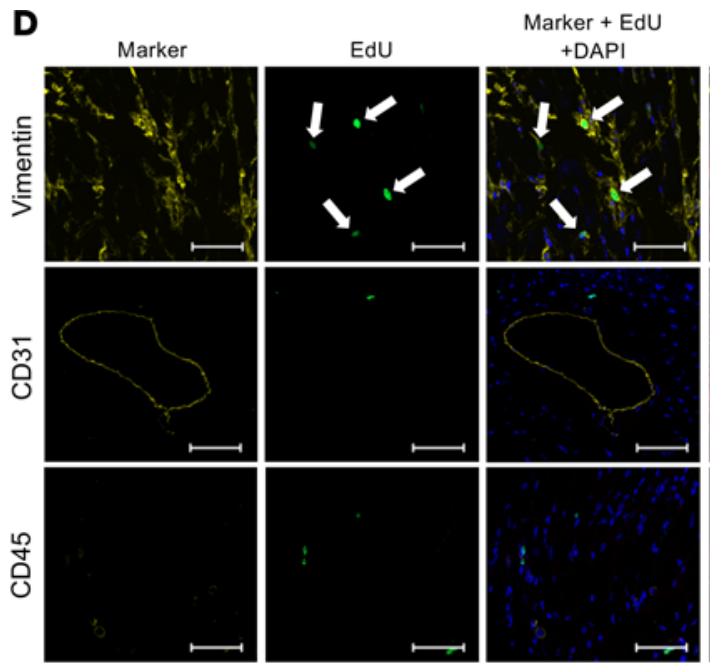

E

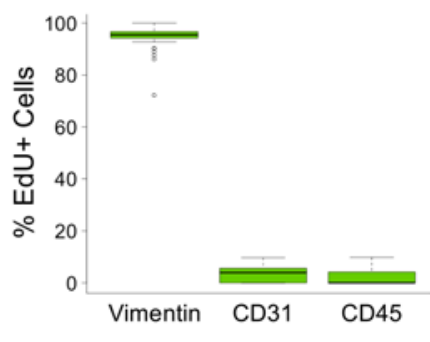

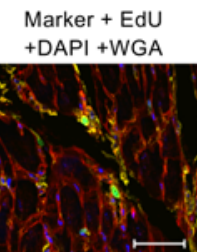

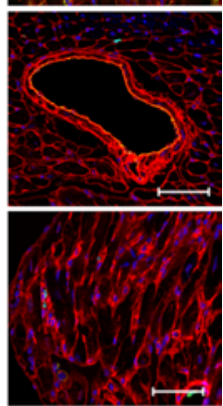

Figure 6. BET inhibition prevents cardiac fibroblast proliferation. (A) Experimental protocol ( $n=4$ mice/treatment group). (B) PLN ${ }^{R 9 C}$ vehicle-treated mice had 3.7-fold more proliferating non-myocytes than WT mice. This was substantially reduced by JQ1. Boxes show IQR; notches, SD; whiskers, 1.5× IQR; circles, extreme outliers. Student's 2-tailed $t$ test. (C) Representative confocal images showing staining for EdU, DAPI, WCA, and cardiac troponin I (cTnl), and merged image. Scale bars: $50 \mu \mathrm{m}$. (D) Representative images from a multimarker analysis demonstrating costaining of EdU with vimentin (arrows) but not with CD31 or CD45. Scale bars: $50 \mu \mathrm{m}$. (E) Quantification of cells staining for both EdU and lineage-specific marker identified the majority of EdU cells as cardiac fibroblasts. Slides were costained with DAPI and WGA ( $n=4$ /group). Boxes show IQR; whiskers, $1.5 \times$ IQR; circles, extreme outliers.

BETs interact with $N F-\kappa B$ in cardiac fibroblasts. The mechanisms by which BETs preferentially suppress inflammatory gene expression in this model of chronic DCM remain unknown. BRD4 is necessary for $\mathrm{NF}-\kappa \mathrm{B}-$ mediated inflammatory gene expression in models of atherosclerosis (22), graft-versus-host disease (23), and cancer $(18,24,25)$. Thus, we investigated whether BETs exert their potent effects on non-myocyte gene expression via NF- $\kappa$ B. Our extensive RNA-Seq studies revealed that NF- $\mathrm{B}$ signaling was strongly upregulated in $\mathrm{PLN}^{\mathrm{R} 9 \mathrm{C}}$ hearts and isolated cardiac non-myocytes at all disease stages, and genes activated by NF- $\mathrm{kB}$ were strongly upregulated in cardiac non-myocytes but not cardiomyocytes (Table 4 and Figure 8A). GSEA in $\mathrm{PLN}^{\mathrm{R} 9 \mathrm{C}}$ vehicle- or JQ1-treated mice against genes known to be directly induced by NF- $\mathrm{B}$ (derived from the Boston University-curated NF- $\mathrm{B}$ target gene list; https://www.bu.edu/nf-kb/gene-resources/target-genes/) showed striking enrichment of $\mathrm{NF}-\mathrm{\kappa B}$ target genes in $\mathrm{PLN}^{\mathrm{R} 9 \mathrm{C}}$ vehicle-treated hearts and substantial attenuation of these genes with longitudinal JQ1 treatment (Figure 8, B and C). These data were confirmed in other curated NF- $\mathrm{NB}$ gene sets, including genes induced by transgenic overexpression of the upstream NF- $\mathrm{B}$ activator IKK $\beta$ in the heart, which induces an inflammatory cardiomyopathy (Supplemental Figure 7) (26).

Acetylation of the RELA subunit of NF- $\mathrm{BB}$ is a well-established marker of increased NF- $\mathrm{B}$ activity (27). We identified markedly increased acetylated lysine 310 of RELA (aK310-RELA) in PLN ${ }^{R 9 C}$ hearts with DCM (Supplemental Figure 8). This finding was independent of JQ1 treatment (Figure $8 \mathrm{D})$, suggesting that NF- $\mathrm{KB}$ activation occurs upstream of BET action, as was previously demonstrated in endothelial cells (22). Next, we performed co-IP of BRD4 with RELA and aK310-RELA, demonstrating a clear physical association in $\mathrm{PLN}^{\mathrm{R} 9 \mathrm{C}}$ mice with DCM, an association that was abolished by treatment with JQ1 (Figure 8E). These data reveal that in this model of chronic progressive DCM,

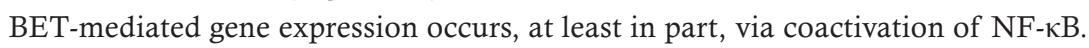


A

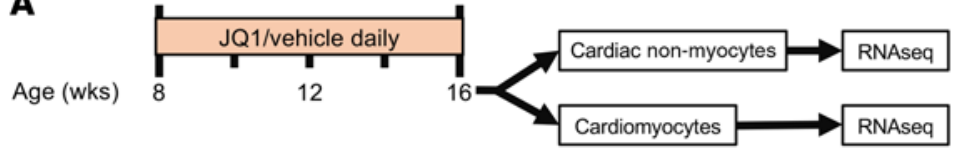

B
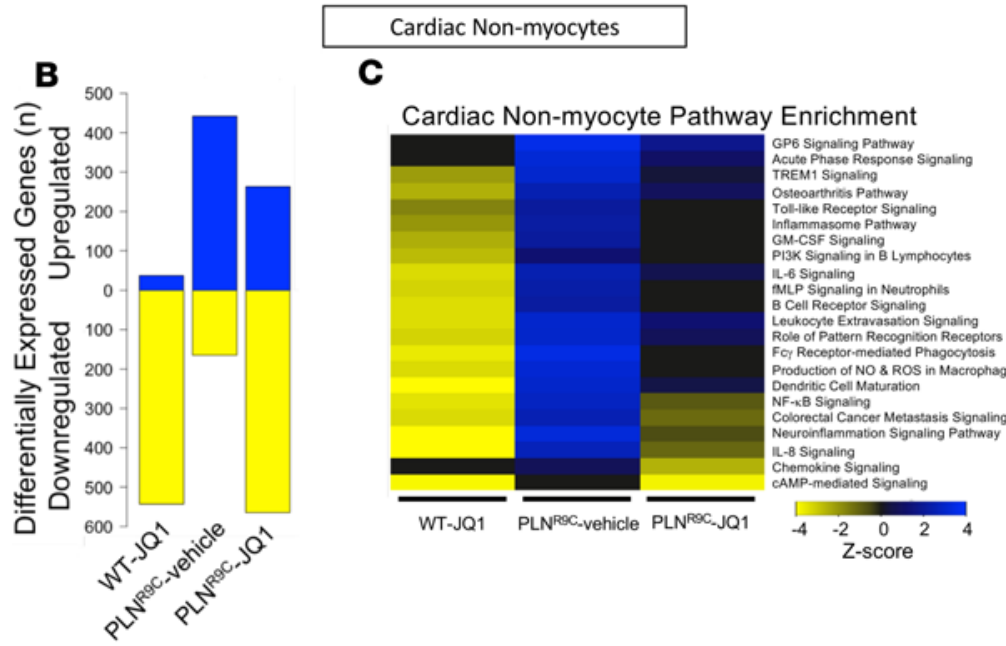

Figure 7. BETs primarily affect cardiac non-myocyte gene expression programs. (A) Experimental protocol ( $n=3$ mice/ treatment group). Non-myocytes: (B) Differentially expressed genes from pooled cardiac non-myocytes (relative to WT vehicle-treated mice) were predominantly downregulated in WT JQ1-treated mice and upregulated in PLN ${ }^{R 9 C}$ vehicle-treated mice. Non-myocytes from PLN ${ }^{R 9 C}$ mice treated with JQ1 displayed a marked shift in gene expression. (C) Pathways enriched in non-myocytes from PLN ${ }^{R 9 C}$ vehicle-treated mice with $Z$ score \pm 2 were all downregulated or not enriched in mice treated with JQ1. Data represent the $Z$ score versus WT vehicle-treated mice. (D) JQ1 treatment shifted gene expression in these pathways from predominantly up- to predominantly downregulated. Cardiomyocytes: (E) The pattern of differentially expressed genes from pooled cardiomyocytes (relative to WT vehicle-treated mice) was similar in PLN ${ }^{R 9 C}$ vehicle-treated and JQ1-treated mice. Further, JQ1 had virtually no effect on gene expression in WT cardiomyocytes. (F) Heat map of genes differentially expressed in PLN ${ }^{\mathrm{RPC}}$ vehicle-treated cardiomyocytes showing little to no change in expression levels with JQ1 treatment. Data plotted are natural log of fold change values versus WT vehicle-treated mice.

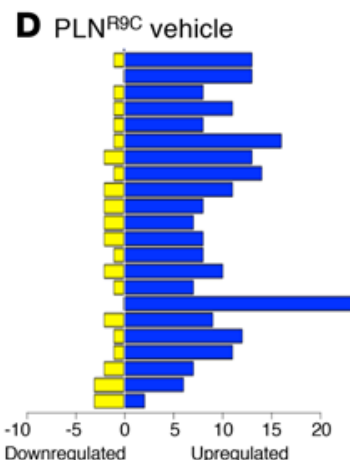
Downregulated Upregulated
Percent Pathway Genes

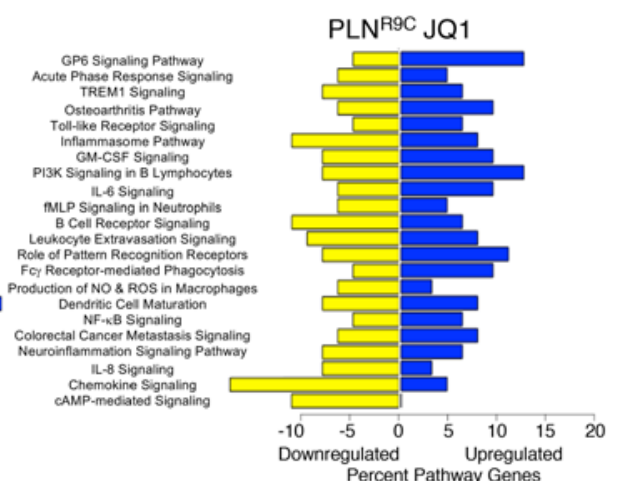

Percent Pathway Genes

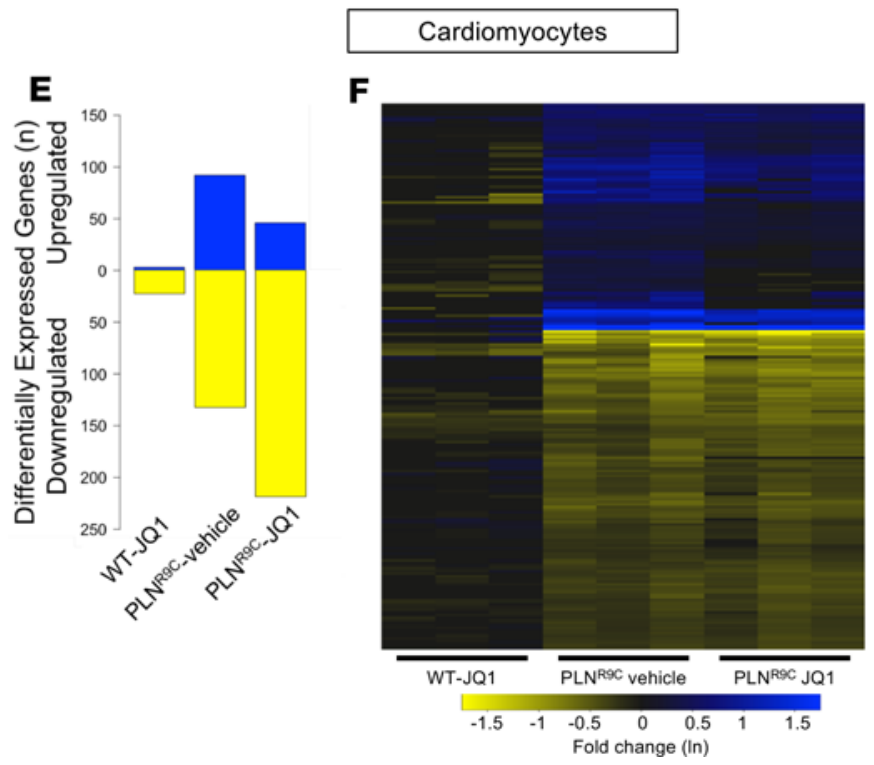


Table 3. Cardiomyocyte pathway enrichment by treatment group

\begin{tabular}{|c|c|c|}
\hline Ingenuity canonical pathway & PLN $^{\mathrm{RgC}}$-vehicle & PLN ${ }^{R 9 C}-J Q 1$ \\
\hline Fatty acid $\beta$-oxidation I & $7 \times 10^{-8}$ & $8 \times 10^{-9}$ \\
\hline Valine degradation I & $2 \times 10^{-3}$ & 0.01 \\
\hline Calcium signaling & $2 \times 10^{-3}$ & 0.01 \\
\hline Leucine degradation I & $2 \times 10^{-3}$ & $5 \times 10^{-3}$ \\
\hline Glycolysis I & $2 \times 10^{-3}$ & NS \\
\hline Gluconeogenesis I & $2 \times 10^{-3}$ & 0.02 \\
\hline Ketogenesis & $2 \times 10^{-3}$ & $5 \times 10^{-3}$ \\
\hline Mevalonate pathway I & $4 \times 10^{-3}$ & $8 \times 10^{-3}$ \\
\hline 4-Aminobutyrate degradation I & $5 \times 10^{-3}$ & $8 \times 10^{-3}$ \\
\hline Isoleucine degradation I & $5 \times 10^{-3}$ & $9 \times 10^{-3}$ \\
\hline Glutaryl-CoA degradation & $5 \times 10^{-3}$ & 0.01 \\
\hline Mitochondrial L-carnitine shuttle pathway & $6 \times 10^{-3}$ & $9 \times 10^{-5}$ \\
\hline
\end{tabular}

Table 4. NF- $K B$ signaling pathway enrichment by treatment group

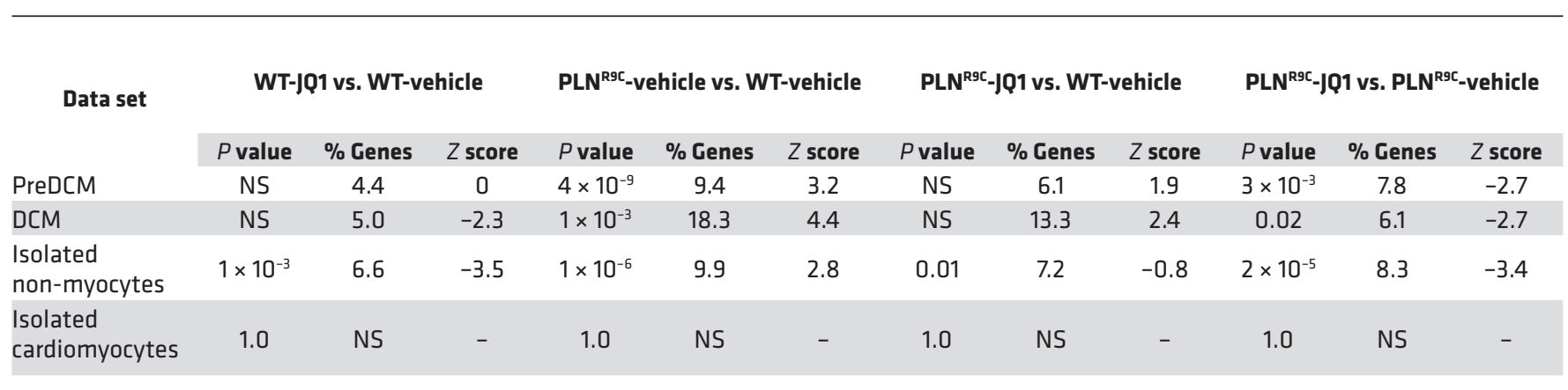

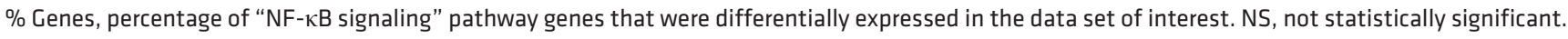

\section{Discussion}

DCM remains a major cause of morbidity and mortality in the United States. Here, we leveraged a well-characterized murine model of insidiously progressive human genetic DCM to demonstrate that BET bromodomain inhibition is an effective strategy for disrupting the progression of chronic nonischemic HF. Unbiased transcriptomic analyses across multiple time points of disease revealed that BETs control a pathologic gene expression program that is dominated by inflammatory and profibrotic signaling networks in the heart. Longitudinal treatment with JQ1 delayed the onset and progression of DCM, revealing that inflammation plays a central role in DCM progression. Importantly, we discovered that BET inhibition has profound and specific effects on fibroblast activation and gene expression, with minimal concomitant effects in cardiomyocytes in this model. Finally, we identified an important mechanistic link between the BET family member BRD4 and NF- $\mathrm{BB}$ in cardiac fibroblasts. Collectively, these data highlight the central role of BETs in DCM pathobiology and define a critical role for BETs in cardiac fibroblast activation. The results establish BET inhibition specifically, and the targeting of inflammatory gene transcription in general, as a potential novel therapeutic strategy for genetic DCM.

BET inhibition with JQ1 reduces myocardial hypertrophy and fibrosis induced by phenylephrine or thoracic aortic banding, and attenuates HF after myocardial infarction (13-15). These prior studies have collectively demonstrated potent suppression of gene networks involved in inflammation, fibrosis, and hypertrophic growth. We have extended these findings to a model of human genetic DCM. The PLN ${ }^{\mathrm{R} 9 \mathrm{C}}$ mouse develops progressive DCM in early-adult life. Importantly, in contrast to preclinical models of acute cardiac stress, $\mathrm{PLN}^{\mathrm{R} 9 \mathrm{C}}$ is a model of insidiously progressive DCM that mimics the tempo of $\mathrm{HF}$ that is typically seen in humans. BET inhibition with JQ1 slowed the progression of DCM, prevented fibrosis, and conferred a survival advantage in $\mathrm{PLN}^{\mathrm{R} 9 \mathrm{C}}$ mice. These findings are particularly relevant to 
A

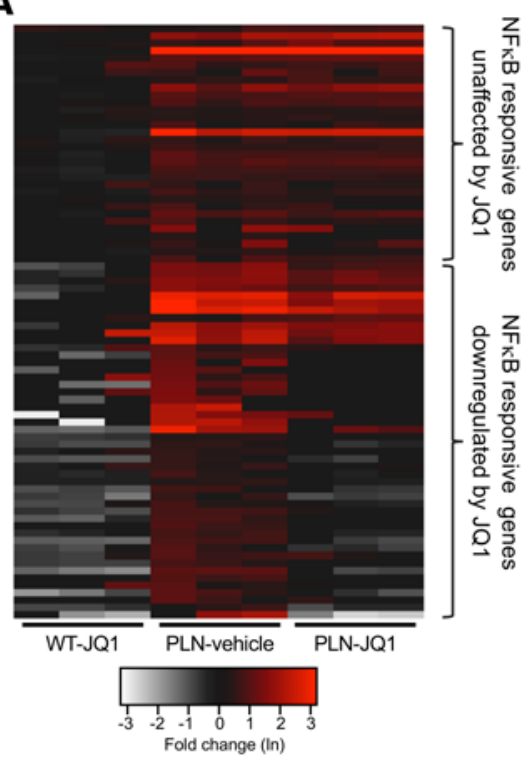

D

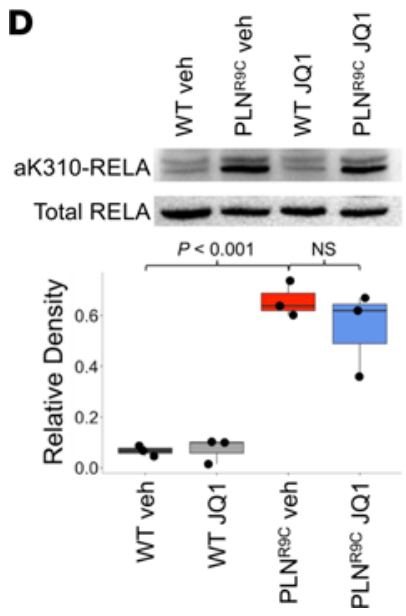

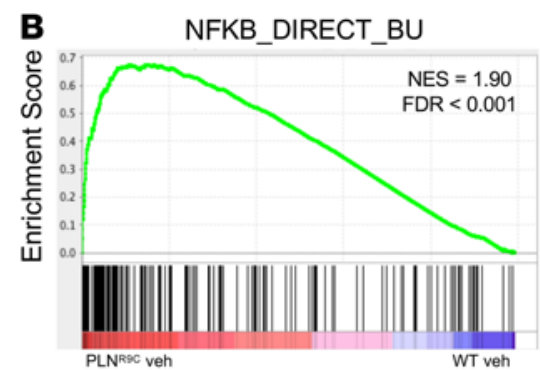

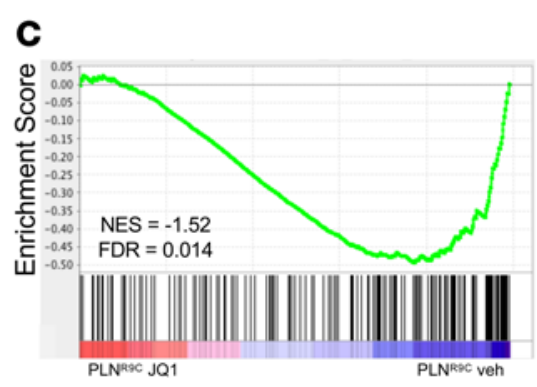

E

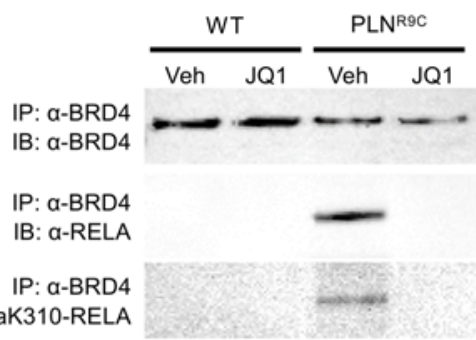

\section{IB: $\alpha$-aK310-RELA}

Figure 8. NF-KB is activated in a BRD4-dependent manner in PLN ${ }^{\mathrm{RIC}}$ hearts. (A) Heat map of genes known to be activated by NF- $\kappa B$ demonstrated uniform upregulation in cardiac nonmyocytes, with a majority of genes being switched off by JQ1 therapy. Data plotted are natural log of fold change values versus WT vehicle-treated mice. CSEA for genes induced (B) in vehicle-treated or attenuated (C) in PLN ${ }^{R 9 C}$ JQ1-treated mice against a curated set of genes known to be directly induced by NF-KB. (D) Western blot showing increased aK310-RELA in PLN ${ }^{R 9 C}$ compared with WT hearts irrespective of JQ1 treatment status (representative images of $n=3$ experiments). Boxes show IQR and whiskers, 1.5x IQR; individual data points are plotted. Student's 2-tailed $t$ test. (E) Western blots (IB) from the co-IP of BRD4 with anti-BRD4, anti-RELA, and anti-ak310-RELA demonstrated direct binding of BRD4 and NF- $K B$ in PLN ${ }^{R 9 C}$ hearts with DCM. This was completely ablated by treatment with JQ1 (representative images of $n=3$ experiments).

human $\mathrm{HF}$, a disease process that, despite intensive research for decades, still leaves the vast majority of individuals with some degree of functional limitation and carries a 5 -year mortality in excess of 50\% (1). Further, current HF pharmacotherapies (neurohumoral blockade, selected vasodilator regimens, and now possibly SGLT-2 inhibitors) stem from our understanding of the systemic consequences of HF. New strategies focused on disrupting disease-specific pathways intrinsic to the heart are urgently needed.

We have previously shown early and progressive activation of inflammatory gene networks in the PLN ${ }^{R 9 C}$ non-myocyte population (3). Interestingly, despite exclusive expression of the PLN ${ }^{\mathrm{R} 9 \mathrm{C}}$ transgene in cardiomyocytes, activation of the cardiac non-myocyte population precedes fibrosis, LV remodeling, and overt cardiomyocyte pathology. Hence, this inflammatory milieu is a secondary consequence of early cardiomyocyte changes that is triggered by an as-yet-undefined mechanism, leaving open the question of whether these changes were truly driving DCM progression. We have shown that BETs are critical mediators of inflammatory gene network expression in DCM, thereby demonstrating a causal role for inflammatory gene network activation in DCM progression. This effect is enriched in the cardiac fibroblast population, consistent with other work that has identified a central role for BRD4 in rat cardiac fibroblast activation (28). Our data also revealed that BETs regulate fibroblast proliferation and myofibroblast activation. As myofibroblasts in the heart are almost exclusively derived from resident cardiac fibroblasts (21), we conclude that BETs likely play a central role in the transdifferentiation of resident cardiac fibroblasts to myofibroblasts in PLN ${ }^{\mathrm{R} 9 \mathrm{C}}$ hearts, marking BETs as nodal mediators of fibroblast activation in chronic DCM.

To extend these findings, we also performed RNA-Seq on pooled cardiomyocytes. Previous work using neonatal rat ventricular cardiomyocytes or human induced pluripotent stem cell-derived cardiomyocytes 
(a cell type that assumes an immature gene expression profile) treated with hypertrophic agonists demonstrated dynamic changes in cardiomyocyte gene expression $(15,29)$. However, in vivo gene signatures from these same studies were still dominated by inflammatory and myofibroblast-enriched gene programs. In our model, we found virtually no gene expression changes triggered by JQ1 in isolated adult cardiomyocytes from WT hearts. Further, the vast majority of cardiomyocyte genes differentially expressed in PLN ${ }^{\mathrm{R} 9 \mathrm{C}}$ JQ1-treated mice were not substantially affected by JQ1. For instance, the predominant gene expression programs affected in DCM cardiomyocytes are metabolic networks (3). We confirmed this shift in metabolic gene expression in PLN ${ }^{\mathrm{R} 9 \mathrm{C}}$ vehicle-treated cardiomyocytes. Yet we observed minimal changes to this metabolic gene program after JQ1 therapy. These data suggest that BETs play a more prominent role in regulating the stress responses of embryonic and/or neonatal cardiomyocytes compared with adult cardiomyocytes. Understanding the cardiomyocyte-specific role of BRD2, BRD3, and BRD4 in vivo using conditional approaches is an important area of future investigation.

The detailed mechanisms by which BETs exert their effect on inflammatory gene transcription in cardiac fibroblasts and other non-cardiomyocyte cell populations are steadily taking shape, though the picture remains incomplete. BETs bind to acetylated lysine residues on histone tails at promoters of active genes. The binding of BRD4, specifically, recruits the positive transcription elongation factor b complex, which promotes pause release of RNA polymerase II, facilitating gene transcription (13, 30, 31). BRD4 also activates gene expression by binding to acetylated lysine residues on non-histones, including the p65/RelA subunit of NF- $\mathrm{NB}(18,24,32)$. In the myocardium, we have shown a direct mechanistic link between BRD4 and acetylated RELA, demonstrating that BRD4 exerts its effects on gene expression, at least in part, via NF-kB. In endothelial cells, cytokine treatment triggers reorganization of chromatin activators from basal endothelial cell enhancers to inflammatory super-enhancers (SEs), which are cis-regulatory elements that permit dense clustering of DNA-bound transcription factors and are associated with exceedingly high levels of transcriptional coactivators $(33,34)$. In endothelial cells, this reorganization activates inflammatory gene expression via NF- $\kappa \mathrm{B}$ in a BRD4-dependent manner (35). Importantly, these authors elegantly demonstrated that NF- $\kappa \mathrm{B}$ activation and SE binding appear to occur upstream of BRD4, as NF- $\mathrm{KB}$ was still bound to SEs in the absence of BRD4. We posit that JQ1 disrupts the ability of NF- $\mathrm{KB}$ to recruit BRD4 to stress-activated SEs in PLN ${ }^{\mathrm{R} 9 \mathrm{C}}$ fibroblasts, limiting changes in gene expression. While this hypothesis requires validation in our model, if correct, it would explain why we did not see a significant decrease in aK310-RELA in PLN ${ }^{\mathrm{R} 9 \mathrm{C}}$ JQ1-treated hearts relative to vehicle in spite of drastic changes in gene expression and cardiac phenotype.

Our data add an important element to the BET story in the heart, yet many key questions remain. While this study contributes to mounting evidence that implicates BRD4 in pathologic cardiac gene expression, the roles of BRD2 and BRD3 in the heart have not been defined. In cultured rat cardiac fibroblasts, $B r d 2$ inhibition did result in reduced expression of myofibroblast markers in response to cytokine stimulation, suggesting BRD2 also has some role in fibroblast gene expression (28). Further, in immune cells, BRD2 binds to the chromatin insulator CTCF; this facilitates SE formation, which in turn recruits BRD4, driving gene expression and cell state transitions via coordinate BRD2-BRD4 interactions at the newly commissioned SEs (36). Whether such a role exists for BRD2 in cardiac fibroblasts remains unknown. Little is known of the biologic function of BRD3, though recent evidence suggests it regulates ribosome biogenesis via control of rRNA production (37). Conditional in vivo gene deletion approaches in specific cardiac cellular compartments will be useful to better define the role of each isoform. Such analyses will complement studies that use small molecule BET bromodomain inhibitors, which bind BRD2, BRD3, and BRD4 (12), though we note that reversibly displacing BETs via a chemical inhibitor will elicit effects very different from gene deletion or depletion.

This study has several limitations. First, mRNA transcript levels do not always correlate with protein levels or protein activity and are thus an indirect measure of pathway activation in vivo. Despite normalization of RNA libraries, the demonstrated proliferation of cardiac fibroblasts confounds the precise interpretation of gene transcript changes among non-myocyte genes in bulk RNA-Seq experiments from whole LV samples. This limitation is partially mitigated by our use of pooled cell fractions, which generate rather pure non-myocyte and cardiomyocyte fractions. However, the non-myocyte fraction, while predominantly consisting of cardiac fibroblasts, does contain other cardiac interstitial cells. More precise techniques such as single-cell RNA-Seq are needed to provide definitive conclusions regarding cardiac fibroblast gene expression. Further, pathway analysis tools are inherently limited by the existing knowledge base for a 
given gene. Finally, while our co-IP studies clearly implicate NF- $\mathrm{B}$ in BRD4-mediated pathologic gene expression changes in the myocardium, we cannot conclusively link this to dynamic reorganization of the transcriptional apparatus at cardiac fibroblast SEs based on these studies.

Fundamentally, $\mathrm{HF}$ is a disease of dysregulated gene expression, much like cancer. However, the mechanisms are distinct: in cancer, the progressive accumulation of mutations drives pathologic gene transcription that ultimately lead to dysregulated cell growth; whereas in HF, the continuous activation of stress signaling networks converges on the transcriptional machinery to alter the myocardial cellular phenotype, ultimately hampering cardiac function. Current HF treatments that target these systemic, upstream stress signaling activators are effective, but morbidity still remains exceedingly high. Consequently, there is an urgent unmet clinical need to directly target intramyocardial processes to improve outcomes (38). Upstream stress signaling networks converge on BET bromodomain proteins, which integrate these signals into specific and distinct gene expression programs. Thus, BET inhibition is a particularly appealing therapeutic strategy that could be both additive to neurohumoral blockade and widely applicable to HF of varying etiologies. These data have extended our understanding of cardiac BET biology and proved the effectiveness of BET inhibition in a model of chronic DCM that closely mimics human HF. Concurrent with ongoing trials in cancer, this knowledge should serve as a springboard for advancing BET inhibition and other chromatin-based therapeutic strategies for the treatment of HF.

\section{Methods}

Mouse model and drug treatment. Mice were housed in ventilated racks in a temperature- and humidity-controlled, pathogen-free facility with 12-hour light/12-hour dark cycles and free access to water and standard laboratory mouse chow. Transgenic mice overexpressing $\mathrm{PLN}^{\mathrm{R} 9 \mathrm{C}}$ under control of the $\alpha-$ cardiac myosin heavy chain promoter in the FVB genetic background have been characterized (4). Male PLN ${ }^{R 9 C}$ mice were compared with age-matched WT male FVB control mice.

The primary end point of mouse survival was defined as survival free of overt HF or death. PLN ${ }^{\text {R9C }}$ mice ultimately develop HF (increased respiratory rate, decreased activity and ability to withdraw from touch, cachexia, and cool skin) in the final stages of disease. Mice typically die within 5 days of symptom onset, marking these symptoms as a surrogate for impending death such that mice were sacrificed to prevent unnecessary pain or suffering.

Stock JQ1 was prepared as previously described (13). Mice were treated with JQ1 (50 mg/kg) or vehicle (10\% DMSO in 10\% hydroxypropyl $\beta$-cyclodextrin) daily via intraperitoneal injection. The length of dose administration varied depending on the study question. Hearts were harvested under deep anesthesia, and LV tissue was extracted for experiments as detailed in Supplemental Methods.

Echocardiography. Mice were anesthetized using an isoflurane vaporizer and attached to ECG leads on a Vevo Mouse Handling Table. Chest hair was removed with depilatory cream (Nair). Transthoracic echocardiography was performed with heart rate $>500$ bpm using a Vevo 770 High-Resolution In Vivo Micro-Imaging System and RMV 707B scan-head. A single experienced echocardiographer blinded to genotype acquired the images and analyzed the data. Parasternal 2D images and M-mode images were acquired to assess (i) LVEDD, (ii) LVESD, (iii) LVWT, which is the combined anterior and posterior wall thickness, and (iv) FS [calculated as FS = (LVEDD - LVESD)/LVEDD)].

Histochemistry. Ventricular tissue was fixed in 4\% paraformaldehyde and paraffin embedded, and sections were cut from apex to base to cover all regions of the LV (see Supplemental Methods for additional details on all histochemical protocols). Nuclei were stained with DAPI (MilliporeSigma, D9542), and the extracellular matrix was stained with anti-wheat germ agglutinin (WGA; Molecular Probes, W32464). To quantify fibrosis, sections were stained with Masson's trichrome, and the ratio of fibrotic area to total ventricular area was measured using a Keyence BZ-X700 microscope.

To assess cell proliferation, mice were injected with EdU. To our knowledge, the optimal dose of EdU for cardiac studies has not been determined. Thus, we performed a dose ranging assay (Supplemental Figure 9), and moved forward with a dose of $25 \mathrm{mg} / \mathrm{kg}$ body weight in $10 \%$ DMSO-PBS for experimental studies. Mice were injected intraperitoneally on 2 consecutive days before sacrifice at 12 weeks of age. Tissue was stained using the Click-iT assay (Thermo Fisher Scientific) and confocal microscopy performed to quantify EdU-positive cells. To identify the non-myocyte cells that were EdU-positive, additional slides were costained with the Click-iT assay and anti-vimentin (Abcam, ab92547), anti-CD31 (Abcam, ab124432), or anti-CD45 (Abcam, ab10558). 
Cell isolation. Myocyte and non-myocyte cell populations were isolated from $\mathrm{PLN}^{\mathrm{R} 9 \mathrm{C} /+}$ and age-matched WT mice treated with either JQ1 or vehicle at 16 weeks of age ( $n=3 /$ treatment group). Hearts were excised, and retrograde coronary perfusion was established via aortic cannulation (39). The heart was perfused with enzyme buffer (see Supplemental Methods) for 10 minutes. The atrium and right ventricle were removed, and the LV was minced into small pieces in transfer buffer (perfusion solution plus $5 \mathrm{mg} / \mathrm{mL} \mathrm{BSA}$ ) and then passed several times through a sterile pipette. The resulting cell suspension was passed through a mesh filter into a $50 \mathrm{~mL}$ centrifuge tube and incubated for 15 minutes at room temperature to allow myocytes to pellet by gravity. The pellet was collected as a cell fraction enriched in myocytes. The supernatant from the filtered cell solution was centrifuged at $370 \mathrm{~g}$ for 5 minutes at $4^{\circ} \mathrm{C}$, and then plated in $75 \mathrm{~mm}$ tissue culture dishes. After 2 hours, dead cells were washed off with $1 \times$ PBS and plated cells collected, resulting in a non-myocyte cell population that was enriched for cardiac fibroblasts (Supplemental Figure 10).

$R N A$-Seq. RNA was extracted from LV tissue or pooled cardiac fibroblast or cardiomyocyte fractions using TRIzol. Prior to cDNA synthesis, 2 rounds of poly(A) selection were performed using oligo-dT Dynabeads (Invitrogen). cDNA libraries were constructed from individual mouse samples, and RNA-Seq libraries were constructed using the Nextera XT DNA Library Preparation Kit (Illumina). Libraries were sequenced on the Illumina platform, then aligned to the mouse reference sequence mm10 using STAR aligner software (40) and a custom data processing pipeline as previously described (3). Data for all RNASeq experiments were deposited in the NCBI's Gene Expression Omnibus (GEO GSE152005)

Bioinformatics pipeline for pathway analysis. Genes were defined as differentially expressed if they met all of the following 3 criteria: (i) normalized read count $>1$ copy/million reads in the RNA-Seq library, (ii) fold-change $>1.33$ (upregulated) or $<-1.33$ (downregulated), and (iii) $P$ value $<0.001$ for the comparison of normalized read counts between experimental samples of interest. To increase stringency, all genes identified as differentially expressed had to meet all of these parameters for all comparisons between $n=3$ mice in each group (total of 9 comparisons per gene). Differentially expressed genes meeting these parameters were identified using a custom pipeline in $\mathrm{R}$ (version 3.0.1). Culled lists of differentially expressed genes were then subjected to bioinformatics analyses using ingenuity pathway analysis (IPA) as described in detail in Supplemental Methods. GSEA was run on expression data preranked by $\log _{2}$ fold change as described previously (15).

Western blotting and immunoprecipitation. Total protein was extracted using RIPA buffer, quantified, separated by SDS-PAGE on 4\%-12\% precast Bis-tris gels and transferred to PVDF membranes. Western blotting was performed using antibodies to BRD2, BRD3, BRD4, vimentin, RELA, and aK310-RELA as described in Supplemental Methods.

IP was performed by targeting BRD4 and the NF- $\mathrm{kB}$ subunit p65/RELA. Freshly harvested whole LVs were dissolved in lysis buffer ( $50 \mathrm{mM}$ Tris- $\mathrm{HCl}, 250 \mathrm{mM} \mathrm{NaCl}, 1 \% \mathrm{NP}-40,1 \mathrm{mM}$ EDTA), and $1 \mathrm{mg}$ protein lysate was subjected to IP with $5 \mu$ g anti-BRD4 antibody (Abcam, ab128874) or anti-NF-kB p65 antibody (Abcam, ab16502) overnight at $4^{\circ} \mathrm{C}$ with rotation. Protein $\mathrm{G}$ magnetic beads $(50 \mu \mathrm{L})$ were added to the protein slurry and incubated for 3 hours with rotation. Beads were then washed twice in lysis buffer, followed by elution in Laemmli buffer. SDS-PAGE and immunoblotting was performed as described above and using

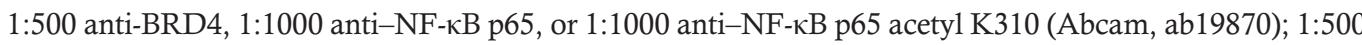
VeriBlot (Abcam, ab131366) was used as the secondary antibody for all IP detection experiments.

Statistics. Data are presented as mean \pm SD. For echocardiography data, time-dependent between-groups variance was calculated using ANOVA with a model-based fixed-effects standard error method. To estimate sample size, a power calculation was performed using the "pwr" package in R based on our prior echo data in $\mathrm{PLN}^{\mathrm{R} 9 \mathrm{C}}$ mice (3). To have $80 \%$ power to detect a $50 \%$ improvement in FS and LVEDD (a moderate effect size) with 4 groups of mice at a significance level of 0.05 , we needed $n=12$ mice/group. Since PLN ${ }^{\mathrm{R} 9 \mathrm{C}}$ mice can die suddenly at any point, we used $n=14 \mathrm{PLN}^{\mathrm{R} 9 \mathrm{C}}$ mice in each arm. As JQ1 was not expected to alter echocardiographic parameters in WT mice, we reduced these arms to $n=7$. Tests of the 4 a priori echocardiographic hypotheses were conducted using Bonferroni's corrected $P$ value for significance (i.e., FDR $<0.0125$.

Survival data were analyzed using the log-rank test; $P$ values less than 0.05 were considered significant. Between-groups differences were calculated using a 2-tailed Student's $t$ test for single paired comparisons where indicated; $P$ values less than 0.05 were considered significant. A FDR less than 0.25 was considered significant for GSEA (41). A Bayesian $P$ value was calculated for RNA-Seq data as described previously (3). Fold change $P$ values less than 0.001 were considered significant for RNA-Seq data. For bioinformatics analyses, $P$ values were corrected for multiple hypothesis testing using the IPA software, and a FDR less than 0.01 was considered significant. See Supplemental Methods for more details on the statistical analyses provided by IPA software. 
Study approval. All studies were approved by the Institutional Animal Care and Use Committees of Harvard Medical School and Emory University, and performed in accordance with the Guide for Care and Use of Laboratory Animals (National Academies Press, 2011).

\section{Author contributions}

AA, HW, ZJ, and JMG conducted experiments, and acquired and analyzed data; SRD analyzed data and processed raw fastq files for GEO submission; MEL analyzed data; DAC conducted experiments, and provided reagents; DYL conducted experiments and analyzed data; JQ provided reagents; JGS designed studies; JEB designed studies and provided reagents; JDB designed studies, conducted experiments, provided reagents, and assisted with manuscript preparation; SMH designed studies, analyzed data, and assisted with manuscript preparation; CES designed studies, analyzed, data and assisted with manuscript preparation; MAB designed studies, conducted experiments, acquired and analyzed data, and wrote and revised the manuscript.

\section{Acknowledgments}

This work was supported by the NIH (T32-HL007745 to AA; K08-HL136873 to MAB; and R01 HL080494 to JGS), the John S. LaDue Memorial Fellowship in Cardiology at Harvard Medical School (to MAB), and the Howard Hughes Medical Institute (to CES).

Address correspondence to: Michael A. Burke, 101 Woodruff Circle, Suite 322, Atlanta, Georgia 30322, USA. Phone: 404.712.2690; Email: michael.burke@emory.edu.

1. Virani SS, et al. Heart disease and stroke statistics - 2020 update: a report from the American Heart Association. Circulation. 2020;141(9):e139-e596.

2. Burke MA, Cook SA, Seidman JG, Seidman CE. Clinical and mechanistic insights into the genetics of cardiomyopathy. $J \mathrm{Am}$ Coll Cardiol. 2016;68(25):2871-2886.

3. Burke MA, et al. Molecular profiling of dilated cardiomyopathy that progresses to heart failure. JCI Insight. 2016;1(6):e86898

4. Schmitt JP, et al. Dilated cardiomyopathy and heart failure caused by a mutation in phospholamban. Science. 2003;299(5611):1410-1413.

5. Chien KR. Meeting Koch's postulates for calcium signaling in cardiac hypertrophy. J Clin Invest. 2000;105(10):1339-1342.

6. Hill JA, Olson EN. Cardiac plasticity. N Engl J Med. 2008;358(13):1370-1380.

7. Truszkowska GT, et al. A study in Polish patients with cardiomyopathy emphasizes pathogenicity of phospholamban (PLN) mutations at amino acid position 9 and low penetrance of heterozygous null PLN mutations. BMC Med Genet. 2015;16:21.

8. Fish M, et al. Mutation analysis of the phospholamban gene in 315 South Africans with dilated, hypertrophic, peripartum and arrhythmogenic right ventricular cardiomyopathies. Sci Rep. 2016;6:22235.

9. Ceholski DK, et al. Functional and transcriptomic insights into pathogenesis of R9C phospholamban mutation using human induced pluripotent stem cell-derived cardiomyocytes. J Mol Cell Cardiol. 2018;119:147-154.

10. Papait $\mathrm{R}$, et al. Genome-wide analysis of histone marks identifying an epigenetic signature of promoters and enhancers underlying cardiac hypertrophy. Proc Natl Acad Sci U S A. 2013;110(50):20164-20169.

11. Sayed D, He M, Yang Z, Lin L, Abdellatif M. Transcriptional regulation patterns revealed by high resolution chromatin immunoprecipitation during cardiac hypertrophy. J Biol Chem. 2013;288(4):2546-2558.

12. Filippakopoulos P, et al. Selective inhibition of BET bromodomains. Nature. 2010;468(7327):1067-1073.

13. Anand P, et al. BET bromodomains mediate transcriptional pause release in heart failure. Cell. 2013;154(3):569-582.

14. Spiltoir JI, et al. BET acetyl-lysine binding proteins control pathological cardiac hypertrophy. J Mol Cell Cardiol. 2013;63:175-179.

15. Duan $\mathrm{Q}$, et al. BET bromodomain inhibition suppresses innate inflammatory and profibrotic transcriptional networks in heart failure. Sci Transl Med. 2017;9(390):eaah5084.

16. Nagarajan S, et al. Bromodomain protein BRD4 is required for estrogen receptor-dependent enhancer activation and gene transcription. Cell Rep. 2014;8(2):460-469.

17. Perry MM, Durham AL, Austin PJ, Adcock IM, Chung KF. BET bromodomains regulate transforming growth factor- $\beta$-induced proliferation and cytokine release in asthmatic airway smooth muscle. J Biol Chem. 2015;290(14):9111-9121.

18. Wu X, Qi J, Bradner JE, Xiao G, Chen LF. Bromodomain and extraterminal (BET) protein inhibition suppresses human T cell leukemia virus 1 (HTLV-1) Tax protein-mediated tumorigenesis by inhibiting nuclear factor $\mathrm{\kappa B}(\mathrm{NF}-\mathrm{\kappa B})$ signaling. J Biol Chem. 2013;288(50):36094-36105.

19. Zuber J, et al. RNAi screen identifies Brd4 as a therapeutic target in acute myeloid leukaemia. Nature. 2011;478(7370):524-528.

20. Chang HY, et al. Diversity, topographic differentiation, and positional memory in human fibroblasts. Proc Natl Acad Sci U S A. 2002;99(20):12877-12882.

21. Kanisicak O, et al. Genetic lineage tracing defines myofibroblast origin and function in the injured heart. Nat Commun. 2016;7:12260

22. Brown JD, et al. NF-kB directs dynamic super enhancer formation in inflammation and atherogenesis. Mol Cell. 2014;56(2):219-231.

23. Sun $Y$, et al. BET bromodomain inhibition suppresses graft-versus-host disease after allogeneic bone marrow transplantation in mice. Blood. 2015;125(17):2724-2728.

24. Zou Z, et al. Brd4 maintains constitutively active NF- $\mathrm{kB}$ in cancer cells by binding to acetylated RelA. Oncogene. 2014;33(18):2395-2404. 
25. Kleppe M, et al. Dual Targeting of oncogenic activation and inflammatory signaling increases therapeutic efficacy in myeloproliferative neoplasms. Cancer Cell. 2018;33(1):29-43.e7.

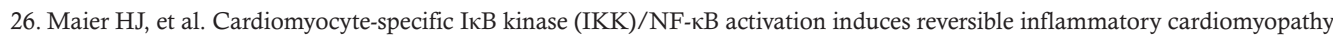
and heart failure. Proc Natl Acad Sci U S A. 2012;109(29):11794-11799.

27. Hayden MS, Ghosh S. Shared principles in NF-kappaB signaling. Cell. 2008;132(3):344-362.

28. Stratton MS, et al. Dynamic chromatin targeting of BRD4 stimulates cardiac fibroblast activation. Circ Res. 2019;125(7):662-677.

29. Stratton MS, et al. Signal-dependent recruitment of BRD4 to cardiomyocyte super-enhancers is suppressed by a MicroRNA. Cell Rep. 2016;16(5):1366-1378.

30. Jang MK, Mochizuki K, Zhou M, Jeong HS, Brady JN, Ozato K. The bromodomain protein Brd4 is a positive regulatory component of P-TEFb and stimulates RNA polymerase II-dependent transcription. Mol Cell. 2005;19(4):523-534.

31. Yang $\mathrm{Z}$, et al. Recruitment of $\mathrm{P}-\mathrm{TEFb}$ for stimulation of transcriptional elongation by the bromodomain protein Brd4. Mol Cell. 2005;19(4):535-545

32. Huang B, Yang XD, Zhou MM, Ozato K, Chen LF. Brd4 coactivates transcriptional activation of NF-kappaB via specific binding to acetylated RelA. Mol Cell Biol. 2009;29(5):1375-1387.

33. Hnisz D, et al. Super-enhancers in the control of cell identity and disease. Cell. 2013;155(4):934-947.

34. Lovén J, et al. Selective inhibition of tumor oncogenes by disruption of super-enhancers. Cell. 2013;153(2):320-334

35. Brown JD, et al. NF-kB directs dynamic super enhancer formation in inflammation and atherogenesis. Mol Cell. 2014;56(2):219-231.

36. Cheung KL, et al. Distinct roles of Brd2 and Brd4 in potentiating the transcriptional program for Th17 cell differentiation. Mol Cell. 2017;65(6):1068-1080.e5.

37. Lambert JP, et al. Interactome rewiring following pharmacological targeting of BET bromodomains. Mol Cell. 2019;73(3):621-638.e17.

38. Gheorghiade M, et al. Developing new treatments for heart failure: focus on the heart. Circ Heart Fail. 2016 ;9(5):e002727.

39. Liao R, Jain M. Isolation, culture, and functional analysis of adult mouse cardiomyocytes. Methods Mol Med. 2007;139:251-262.

40. Dobin A, Gingeras TR. Mapping RNA-seq reads with STAR. Curr Protoc Bioinformatics. 2015;51:11.14.1-11.14.19.

41. Subramanian A, et al. Gene set enrichment analysis: a knowledge-based approach for interpreting genome-wide expression profiles. Proc Natl Acad Sci U S A. 2005;102(43):15545-15550. 